\title{
The discrete fractional order difference applied to an epidemic model with indirect transmission
}

\author{
Carmen Coll, Alicia Herrero*, Damián Ginestar, Elena Sánchez \\ Institut Universitari de Matemàtica Multidisciplinar, Universitat Politècnica de València, Camí de Vera, s/n, València 46022, Spain
}

\section{A R T I C L E I N F O}

\section{Article history:}

Received 23 April 2021

Revised 3 November 2021

Accepted 8 November 2021

Available online 17 November 2021

\section{Keywords:}

Epidemic process

Discrete fractional-order

Indirect transmission

Stability

\begin{abstract}
A B S T R A C T
A discrete fractional order model is proposed to analyze the behaviour of an epidemic process with indirect transmission. This model is based on a discrete version of the GrunwaldLetnikov fractional derivative operator. Some properties of this operator are shown and used to derive a truncated version of the operator, which is used to propose a model with short-term memory. Based on the biological meaning of the problem, some bounds have been obtained to assure the nonnegativity of the model solution. The $(\alpha, k)$-Basic Reproduction Number has been introduced and used to analyze the stability of the solution around its equilibrium points. Moreover, the influence of the fractional order, $\alpha$, and the memory steps, $k$, on the behaviour of the solution has been analyzed. Finally, the results obtained have been clarified by means of numerical simulations of a model for the evolution of an infection by Salmonella in a hens farm.
\end{abstract}

(c) 2021 The Authors. Published by Elsevier Inc. This is an open access article under the CC BY-NC-ND license (http://creativecommons.org/licenses/by-nc-nd/4.0/)

\section{Introduction}

Mathematical models, both in continuous and discrete time, are a useful tool to describe an epidemic process. Most of these models are based on differential equations or difference equations, see, for example, [1-4] and the references there in. However, this type of model only takes into account what happens in the current state without considering the historical states of the process, that is, what has happened in previous states. This implies that they neglect the effect of memory on determining the system outcomes. Models based on fractional derivative approximations can describe long-term or short-term memory processes, allowing a better simulation of the dynamical system. In the last years, several authors have introduced different definitions of fractional derivative (see, for instance, $[5,6]$ ). Particular definitions of fractional derivatives are given, for example, in [7,8], applying them on logistic map and diffusion equation in a Caputo-like delta sense, and in [9], studying fractal processes. Moreover, the physical and geometrical interpretation of some fractional derivatives is analyzed in [10].

The choice of fractional order derivatives in the construction of an epidemic model has certain advantages since infectious diseases are dynamic processes with memory. Thus, the model can be improved using a fractional order derivative since the variables depend not only on the current state but also on all previous historical states [11]. The fact of including short or long-term memory allows us to better understand some mechanisms that influence the outbreak of diseases that can be

\footnotetext{
* Corresponding author::

E-mail addresses: mccoll@mat.upv.es (C. Coll), aherrero@mat.upv.es (A. Herrero), dginesta@mat.upv.es (D. Ginestar), esanchezj@mat.upv.es (E. Sánchez).
} 
provided by parameters neglected if memory is not taken into account. For example, the main features of immune response involve memory, [12]. Moreover, the consideration of memory in the mathematical model is also useful for designing and determining how to implement control strategies.

In the literature, most epidemic models are based on differential equations, including fractional ones. Usually, the analysis of the model consists on applying the discretization process for the continuous-time epidemic model to better assess the effect of smaller and larger time steps on the model behaviour [13]. However, some of the reasons that make to work with discrete systems interesting are based on the fact that, populations have non-overlapping generations, [14], what happens, for example, in infectious deseases and also, statistical data on epidemics are collected at discrete times and, therefore, it is easier to compare these data with the output of a discrete system [15-17].

In recent decades, many papers have appeared in the literature dealing on how to apply the fractional order derivative to the study of epidemic models. Most models, follow the process of starting from models represented by equations or systems of differential equations, and substituting the ordinary derivatives by a fractional order ones using, for example, Caputo's definition of fractional derivative, [6]. A discrete model is obtained by applying some of the known discretization processes for this derivative [18-22]. In the last year, a new notion of fractional G-transform using modified RiemannLiouville derivative with the Mittag-Leffler function as a kernel is introduced to analyze fractional epidemic models, [23].

Similarly as it is done for continuous models, for discrete models the authors start from a discrete model and apply a fractional approximation of the difference operator. Linear discrete-time fractional-order systems are studied in [24] using the Grünwald-Letnikov fractional derivative discrete approximation. In [25] some new results concerning the analysis of stability of discrete-time fractional systems are given. In [26] the practical stability of positive fractional discrete-time systems is analyzed. And, a compendium for fractional-order positive system can be found in [27].

The use of the discrete fractional order operators to study the dynamics of a disease provides us with a more flexible tool to model the disease evolution since the impact of memory on the dynamics is included in the model. In our case, we will use a truncated approximation for a discrete fractional order operator that includes a short-term memory process, this could be interpreted as the inclusion of a set of delays in the model. Biologically, this may represent gestation times, incubation periods or simply consider the time required for a certain process to occur within a complicated biological system, in particular it may represent the time required between the infection of a cell and the production of new viruses. As an example, in the case of a model that reflects the dynamics of the Hepatitis C virus (HCV), the use of a fractional order operator in the model can represent intermediate cellular interactions and intracellular delay of the viral life cycle and incorporate a discrete time-delay to justify short-term memory, [28].

Motivated by the facts exposed above, in this paper, we consider a discrete-time model established from the GrünwaldLetnikov fractional order derivative. Moreover, we are interested on studying epidemic models that include the indirect transmission of the disease due to the underlying contamination in the space where we have the population, (see [3,2933]). That is, individuals can become infected with residues found in their environment. Then, in addition to the susceptible and infected individuals variables, it is considered a variable measuring the amount of contaminant that is found in the environment, which has been generated by infected individuals. In this way, the theory of a fractional difference operator is introduced, a fractional discrete model for infectious disease is proposed and some properties of the model are analyzed.

The rest of this paper is organized as follows: Section 2 presents a formulation of the general epidemic model and a new model that uses the discrete-time fractional-order (DTFO) difference operator [24]. We also introduce some extra notation and show some properties of the DTFO operator coefficients that will be useful throughout the paper. Section 3 addresses the analysis of the stability property of the epidemic model. In Section 4, we analyze the effects of the fractional order and the memory steps on the behaviour of the model. In Section 5, we present some numerical results, corresponding to different cases that show the effect of changing the parameters of the DTFO operator on the epidemic model behaviour. Finally, the conclusions of the paper are summarized in Section 6.

\section{Problem statement}

In the general epidemic model we consider the following conditions:

- The population in the model is distributed in Susceptible individuals $S(t)$ and Infected individuals $I(t)$. And, we consider that the transmission of this disease occurs by contact with the environmental pollutant $C(t)$.

- The Infected individuals remain in the environment and the Contaminant produced by these individuals can infect others individuals.

- The size of the population always remains constant, $P=S(t)+I(t), \forall t \geq 0$, with replacement of dead individuals.

The Infected individuals increase depends on the fraction of Infected individuals over the total population (prevalence of infection). The parameter $\sigma$ represents the exposition rate of Susceptible individuals by contact with Contaminant and the number of new infections (incidence) is given by $\sigma C(t) S(t)$; this term makes the model nonlinear. Under these conditions, the dynamics of the process can be modelled using the following system of difference equations,

$$
\begin{aligned}
& S(t+1)=p S(t)-\sigma C(t) S(t)+\mu(t) P, \\
& I(t+1)=q I(t)+\sigma C(t) S(t), \\
& C(t+1)=s C(t)+\beta I(t), t \geq 0
\end{aligned}
$$


Table 1

Parameters of the model.

\begin{tabular}{ll}
\hline Parameter & Description \\
\hline$\sigma$ & Exposition rate of Susceptible individuals. \\
$\mu(t) P$ & Replacement rate of dead individuals. \\
$p / q$ & Survival rate of the Susceptible and Infected population, respectively. \\
$s$ & Survival rate of the Contaminant. \\
$\beta$ & The amount of Contaminant produced by each infected individual. \\
\hline
\end{tabular}

where the meaning of the parameters is given in Table 1.

We assume the following conditions on the parameters of the model: $0<p, q, s, \sigma<1$ and $\beta \geq 0$. Previous works related with this model can be found in $[2,3,32]$.

System (1) can be rewritten using the following matrix form

$$
x(t+1)=A x(t)+f(x(t))+B \mu(t) P, \quad t \geq 0
$$

with

$$
A=\left(\begin{array}{lll}
p & 0 & 0 \\
0 & q & 0 \\
0 & \beta & s
\end{array}\right)
$$

$f(x(t))=\sigma x_{1}(t) x_{3}(t) v_{1}$ and $B=e_{1}$, being $v_{1}=(-1,1,0)^{T}$ and $e_{1}$ the first vector of the canonical basis for the threedimensional space $\mathbb{R}^{3}$. The vector $x(t)=\left(x_{i}(t)\right)_{i=1,2,3}^{T}$ denotes Susceptible individuals $\left(x_{1}(t)\right)$, Infected individuals $\left(x_{2}(t)\right)$ and Contaminant $\left(x_{3}(t)\right)$. Moreover, we assume $x_{1}(t)+x_{2}(t)=P, \forall t \geq 0$.

We define the first-order difference operator for $x(t+1)$ as $\Delta^{1} x(t+1)=x(t+1)-x(t)$ and, rewriting equation (2), we have

$$
\Delta^{1} x(t+1)=(A-I) x(t)+f(x(t))+B \mu(t) P, \quad t \geq 0 .
$$

Now, our goal is to generalize the previous system by applying a discrete-time fractional order (DTFO) operator to analyze the behavior of the model. For that, we use the following discrete-time fractional order Grünwald-Letnikov operator $\Delta^{\alpha}$ defined with the zero initial time as follows [24] :

$$
\Delta^{\alpha} x(t)=\frac{1}{h^{\alpha}} \sum_{j=0}^{t} a_{j}^{\alpha} x(t-j h),
$$

where the fractional order $\alpha$ satisfies $0<\alpha \leq 1$ and the strictly positive real number, $h \in \mathbb{R}^{+}$, is a sampling period taken equal to unity in all what follows. In this expression

$$
a_{j}^{\alpha}=(-1)^{j}\left(\begin{array}{l}
\alpha \\
j
\end{array}\right), j \geq 0
$$

with

$$
\left(\begin{array}{l}
\alpha \\
j
\end{array}\right)= \begin{cases}1 & j=0 \\
\frac{\alpha(\alpha-1) \ldots(\alpha-j+1)}{j !} & j>0\end{cases}
$$

Using the DTFO operator we propose the following discrete-time fractional order model given by

$$
\Delta^{\alpha} x(t+1)=(A-I) x(t)+f(x(t))+B \mu(t) P, \quad t \geq 0 .
$$

Note that, for $\alpha=1$ we obtain system (4). Now, rewriting system (8) we have

$$
x(t+1)=(A-I) x(t)-\sum_{j=1}^{t+1} a_{j}^{\alpha} x(t+1-j)+f(x(t))+B \mu(t) P, t \geq 0 .
$$

To study this system, we first analyze some properties of the coefficients introduced by the DTFO operator.

\subsection{Properties of DTFO coefficients}

In this subsection, we give some properties of the coefficients, which will be useful through the paper.

Proposition 1. The coefficients $a_{j}^{\alpha}, j \geq 1,0<\alpha \leq 1$, defined in (6), satisfy that

$$
-1<a_{j}^{\alpha}<0 \quad \text { and } \quad\left|a_{j+1}^{\alpha}\right|<\left|a_{j}^{\alpha}\right|<1 .
$$


Proof. From the definition of $a_{j}^{\alpha}$ we have that, if $j \geq 1$ and $0<\alpha \leq 1$,

$$
a_{j}^{\alpha}=(-1)^{j} \frac{\alpha(\alpha-1) \cdots(\alpha-j+1)}{j !}=-\frac{\alpha(1-\alpha) \cdots(j-1-\alpha)}{j !}<0
$$

and hence $a_{j}^{\alpha}>-\frac{\alpha}{j}>-1$.

On the other hand, we also have that $a_{j+1}^{\alpha}=\frac{j-\alpha}{j+1} a_{j}^{\alpha}$ then, since $j-\alpha<j+1$, we get $\left|a_{j+1}^{\alpha}\right|<\left|a_{j}^{\alpha}\right|<1$.

Another quantity that will be useful through the paper is the sum of these coefficients. We denote by

$$
\Gamma_{k}^{\alpha}=1-\Sigma_{k}^{\alpha} \quad \text { with } \quad \Sigma_{k}^{\alpha}=\sum_{j=0}^{k} a_{j}^{\alpha}, \quad k \geq 0,0<\alpha \leq 1
$$

Next proposition gives some properties of this sum of coefficients.

Proposition 2. The sum $\Sigma_{k}^{\alpha}, k \geq 0,0<\alpha \leq 1$ defined in (11), satisfies the following properties:

(i) $\Sigma_{0}^{\alpha}=1$ and $\Sigma_{k}^{1}=0$.

(ii) $0<\Sigma_{k}^{\alpha}=-\frac{(k-\alpha)}{\alpha} a_{k}^{\alpha}<1$, if $k \geq 1$.

(iii) $\Gamma_{k}^{1}=1$ and $\Gamma_{k}^{\alpha}<1$.

Proof. (i) Follows from the definition of $\Sigma_{k}^{\alpha}$.

(ii) First, we show that $\Sigma_{k}^{\alpha}=\frac{(1-\alpha) \ldots(k-\alpha)}{k !}$ by induction on $k$.

For $k=1, \Sigma_{1}^{\alpha}=\Sigma_{0}^{\alpha}+a_{1}^{\alpha}=1-\alpha$. Now, we assume that the property is true for $k-1$, so $\Sigma_{k-1}^{\alpha}=\frac{(1-\alpha) \ldots(k-1-\alpha)}{(k-1) !}$ and we are going to prove it for $k$ :

$$
\Sigma_{k}^{\alpha}=\Sigma_{k-1}^{\alpha}+a_{k}^{\alpha}=\frac{(1-\alpha) \ldots(k-1-\alpha)}{(k-1) !}\left(1-\frac{\alpha}{k}\right)=\frac{(1-\alpha) \ldots(k-\alpha)}{k !}
$$

So, using the expression (10),

$$
\Sigma_{k}^{\alpha}=\frac{k-\alpha}{\alpha} \frac{\alpha(1-\alpha) \ldots(k-1-\alpha)}{k !}=-\frac{k-\alpha}{\alpha} a_{k}^{\alpha} .
$$

Since $a_{k}^{\alpha}<0$ we have $\Sigma_{k}^{\alpha}>0, k \geq 1$. Moreover,

$$
\left|\Sigma_{k}^{\alpha}\right|=\frac{k-\alpha}{\alpha}\left|a_{k}^{\alpha}\right|<\frac{k-\alpha}{\alpha} \frac{\alpha}{k}=\frac{k-\alpha}{k}<1 .
$$

(iii) The result follows directly from the relationship between $\Gamma_{k}^{\alpha}$ and $\Sigma_{k}^{\alpha}$, for all $k \geq 0$ and $0<\alpha \leq 1$.

\subsection{Discrete-time fractional order model}

In the last years, a truncated operator has been used in [25,26] to study the practical stability of a fractional order discrete system. In a similar way, from the properties of the coefficients of the DTFO operator, we observe that their absolute values form a decreasing sequence. Then, at a certain time these coefficients will be negligible. Therefore, we will truncate the definition of the operator to have a model with short-term memory. Thus, we define the truncated operator in $k$ steps, as follows:

$$
\Delta_{k}^{\alpha} x(t)=\sum_{j=0}^{k} a_{j}^{\alpha} x(t-j)
$$

Using this truncated DTFO operator we obtain the discrete-time fractional order model given by

$$
\Delta_{k}^{\alpha} x(t+1)=(A-I) x(t)+f(x(t))+B \mu(t) P, \quad t \geq 0
$$

that is,

$$
x(t+1)=(A-I) x(t)-\sum_{j=1}^{k} a_{j}^{\alpha} x(t+1-j)+f(x(t))+B \mu(t) P, t \geq 0 .
$$

This is a model with $k$ steps of memory since the solution $x(t+1)$ depends on the states $x(t), x(t-1), \ldots, x(t-k+1)$. The condition of constant population $P$ at any time $t \geq 0$ implies that the addition of the two first equations gives

$$
\mu(t) P=(q-p) x_{1}(t)+P\left(1-q+\Sigma_{k}^{\alpha}\right), \quad t \geq 0 .
$$


So, applying this condition to our model (14), it can be rewritten in components as

$$
\begin{aligned}
& \left.x_{1}(t+1)=(q-1+\alpha)\right) x_{1}(t)-\sum_{j=2}^{k} a_{j}^{\alpha} x_{1}(t+1-j)-\sigma x_{1}(t) x_{3}(t)+P\left(1-q+\Sigma_{k}^{\alpha}\right), \\
& x_{2}(t+1)=(q-1+\alpha) x_{2}(t)-\sum_{j=2}^{k} a_{j}^{\alpha} x_{2}(t+1-j)+\sigma x_{1}(t) x_{3}(t), \\
& x_{3}(t+1)=(s-1+\alpha) x_{3}(t)-\sum_{j=2}^{k} a_{j}^{\alpha} x_{3}(t+1-j)+\beta x_{2}(t),
\end{aligned}
$$

or, equivalently,

$$
x(t+1)=\tilde{A} x(t)-\sum_{j=2}^{k} a_{j}^{\alpha} x(t+1-j)+f(x(t))+B\left(1-q+\Sigma_{k}^{\alpha}\right) P, \quad t \geq 0,
$$

where

$$
\tilde{A}=\left(\begin{array}{ccc}
q-1+\alpha & 0 & 0 \\
0 & q-1+\alpha & 0 \\
0 & \beta & s-1+\alpha
\end{array}\right)
$$

We can observe that, in this model, the survival rate of the Susceptible population does not appear explicitly in the equations.

We have to assure that, under the conditions of no infection and no recruiment of population, then both, Infected individuals and Contaminant, disappear. So, in order to get consistency with this particular case, the matrix $\tilde{A}$ has to be a stable matrix, that is, $\rho(\tilde{A})<1$ where $\rho(M)$ denotes the spectral radius of a matrix $M$ [34]. Therefore, from now on, we only consider $\alpha \in \Lambda_{q, s}$, where

$$
\left.\left.\Lambda_{q, s}=\{\alpha \in] 0,1\right] / 0 \leq 1-\alpha<q \text {, and } 0 \leq 1-\alpha<s\right\} .
$$

In the next section, we compute the equilibrium points of this discrete time fractional order system and their stability characteristics.

\section{Stability}

\subsection{Equilibrium points}

As system (16) is a fractional order system with $k$ memory steps, then it has the form $x(t+1)=g(x(t), x(t-1), \cdots, x(t+$ $1-k)$ ), so its equilibrium points will be those points $x^{*}=\left(x_{1}^{*} x_{2}^{*} x_{3}^{*}\right)^{T}$ such that $x^{*}=g\left(x^{*}, x^{*}, \cdots, x^{*}\right)$. For system $(15)$ we have that the disease-free equilibrium point $x_{f}^{*}$ is

$$
x_{1 f}^{*}=P, \quad x_{2 f}^{*}=0, \quad x_{3 f}^{*}=0
$$

for any value $\alpha \in \Lambda_{q, s}$ and $k \geq 1$. Note, that this agrees with the case $\alpha=1$ when the first order difference is considered.

The endemic equilibrium point $x_{e}^{*}(\alpha, k)$ is obtained for $x_{2}^{*} \neq 0$ having that

$$
\begin{gathered}
x_{1 e}^{*}(\alpha, k)=\frac{\left(1-q+\Sigma_{k}^{\alpha}\right)\left(1-s+\Sigma_{k}^{\alpha}\right)}{\sigma \beta}, \\
x_{2 e}^{*}(\alpha, k)=P-x_{1 e}^{*}(\alpha, k), \quad x_{3 e}^{*}(\alpha, k)=\frac{\beta\left(P-x_{1 e}^{*}(\alpha, k)\right)}{1-s+\Sigma_{k}^{\alpha}},
\end{gathered}
$$

where clearly $1-s+\Sigma_{k}^{\alpha} \neq 0$ and $x_{e}^{*}(\alpha, k) \geq 0$ since $\Sigma_{k}^{\alpha}>0$ and $0<q, s<1$.

We summarize the previous reasoning in the following result.

Proposition 3. The DTFO system (16) has two equilibrium points:

(i) The disease-free equilibrium point, $x_{f}^{*}=\left(\begin{array}{lll}P & 0 & 0\end{array}\right)^{T}$.

(ii) The endemic equilibrium point, $x_{e}^{*}(\alpha, k)=\left(x_{1 e}^{*}(\alpha, k) x_{2 e}^{*}(\alpha, k) x_{3 e}^{*}(\alpha, k)\right)^{T}$ given by (18).

Before studying the stability of the DFTO system (16), it is important to assure that our model is a good representation of the epidemiologic process. Taking into account that only nonnegative solutions have biological sense, in the following proposition we establish an upper limit on the size of the population $P$,

$$
P_{u}(\alpha, k)=\frac{q-1+\alpha}{1-q+\Sigma_{k}^{\alpha}} x_{1 e}^{*}(\alpha, k)
$$


in order to assure the nonnegativity condition on the solution.

Proposition 4. If $P<P_{u}(\alpha, k)$, given in (19), then the solution of the DTFO system (16) is nonnegative.

Proof. From $x_{2}(t) \leq P$, for all $t \geq 0$, and the equation of system (15) relating the contaminant and the infection, we have

$$
x_{3}(t+1) \leq(s-1+\alpha) x_{3}(t)-\sum_{j=2}^{k} a_{j}^{\alpha} x_{3}(t+1-j)+\beta P .
$$

Then, we can construct the following system

$$
y(t+1)=H y(t)+\beta P e_{1},
$$

where $y(t)=\left(x_{3}(t) x_{3}(t-1) \ldots x_{3}(t+1-k)\right)^{T}$ and $H$ is a block matrix given by

$$
H=\left(\begin{array}{cccccc}
s-1+\alpha & -a_{2} & -a_{3} & \cdots & -a_{k-1} & -a_{k} \\
1 & 0 & 0 & \cdots & 0 & 0 \\
0 & 1 & 0 & \cdots & 0 & 0 \\
\vdots & \vdots & \vdots & & \vdots & \vdots \\
0 & 0 & 0 & \cdots & 1 & 0
\end{array}\right),
$$

whose solution is $y(t)=H^{t} y(0)+\beta P \sum_{i=0}^{t-1} H^{j} e_{1}$.

Now, we prove that $\rho(H)<1$. The characteristic polynomial of matrix $H$ is given by

$$
|\lambda I-H|=\lambda^{k}-\lambda^{k-1}(s-1+\alpha)+\lambda^{k-2} a_{2}+\cdots+\lambda a_{k-1}+a_{k}=0,
$$

where we have considered that $\alpha \in \Lambda_{q, s}$.

Using a well-known algebraic result on the bounds of the roots of a polynomial, we have that the solutions of the first equation satisfy

$$
|\lambda| \leq \max \left(1, s-1+\alpha-a_{2}-\ldots-a_{k}\right)=\max \left(1, s-\Sigma_{k}^{\alpha}\right)=1 .
$$

We conclude that $s-\Sigma_{k}^{\alpha}<s<1$ using Proposition 2. Moreover, if there exists a solution $\lambda_{0} \in \mathbb{C}$ with $\left|\lambda_{0}\right|=1$ then

$$
1=\left|\lambda_{0}^{k}\right| \leq|s-1+\alpha|+\left|a_{2}\right|+\cdots+\left|a_{k}\right|=s-\Sigma_{k}^{\alpha}<s<1,
$$

which is a contradiction. So, $|\lambda|<1$ for any solution of the equation. Then, we have proved that $\rho(H)<1, I-H$ is an invertible matrix and $\sum_{i=0}^{\infty} H^{j}=(I-H)^{-1}$. Therefore,

$$
\left(x_{3}(t) x_{3}(t-1) \ldots x_{3}(t+1-k)\right)^{T} \leq \beta P(I-H)^{-1} e_{1}=\frac{\beta P}{1-s+\Sigma_{k}^{\alpha}}(1 \ldots 1)^{T} .
$$

From the condition $P<P_{u}(\alpha, k)$, where $P_{u}(\alpha, k)$ is given in (19), we arrive at

$$
x_{3}(t) \leq \frac{q-1+\alpha}{\sigma} .
$$

Hence $q-1+\alpha-\sigma x_{3}(t) \geq 0$, for all $t \geq 0$. Taking into account this result together with the conditions $a_{j}^{\alpha}<0$ and $1-q+$ $\Sigma_{k}^{\alpha}>0$, we can assure that the solution of the system (16) is nonnegative for all nonnegative initial states.

From now on we consider the following set

$$
\Omega_{\alpha, k}=\left\{x \in \mathbb{R}^{3} / x_{1}+x_{2}=P, P<P_{u}(\alpha, k)\right\},
$$

with $P_{u}(\alpha, k)$ given by (19). Note that, if the trajectory of the system (16) is in $\Omega_{\alpha, k}$, the system is well defined and represents an epidemiologic process with nonnegative solutions.

\section{2. $(\alpha, k)$-Basic reproduction number}

Let us consider the linearization of system (16) around the disease-free equilibrium point $x_{f}^{*}=\left(\begin{array}{lll}P & 0 & 0\end{array}\right)^{T}$. Taking $x_{1}(t) x_{3}(t) \approx P x_{3}(t)$, we have that

$$
\hat{x}(t+1)=E \hat{x}(t)-\sum_{j=2}^{k} a_{j}^{\alpha} \hat{x}(t+1-j),
$$


where $\hat{x}(t)=x(t)-x_{f}^{*}$ and the coefficient matrix results

$$
E=\left(\begin{array}{ccc}
q-1+\alpha & 0 & -\sigma P \\
0 & q-1+\alpha & \sigma P \\
0 & \beta & s-1+\alpha
\end{array}\right)
$$

We can analyze the evolution of this system considering the Infected individuals, $\hat{x}_{2}(t)$, and the Contaminant, $\hat{x_{3}}(t)$. So, we have the following linear subsystem:

$$
z(t+1)=\bar{E} z(t)-\sum_{j=2}^{k} a_{j}^{\alpha} z(t+1-j),
$$

where $z(t)=\left(\hat{x}_{2}(t), \hat{x}_{3}(t)\right)^{T}$ and $\bar{E}=\left(\begin{array}{cc}q-1+\alpha & \sigma P \\ \beta & s-1+\alpha\end{array}\right)$.

As system (25) is a $k$-delayed linear system, it is equivalent to the $k$-stacked linear system,

$$
Z(t+1)=\mathcal{E} Z(t)
$$

with a state vector and matrix $Z(t)=(z(t) z(t-1) \ldots z(t+1-k))^{T}$ and $\mathcal{E}$ given by

$$
\mathcal{E}=\left(\begin{array}{cccccc}
\bar{E} & -a_{2}^{\alpha} I & -a_{3}^{\alpha} I & \cdots & -a_{k-1}^{\alpha} I & -a_{k}^{\alpha} I \\
I & 0 & 0 & \cdots & 0 & 0 \\
O & I & 0 & \cdots & 0 & 0 \\
\vdots & \vdots & \vdots & & \vdots & \vdots \\
0 & 0 & 0 & \cdots & I & 0
\end{array}\right)
$$

respectively.

In order to define the basic reproduction number of our system, we use the next generation matrix approach [35]. For that we decompose matrix $\mathcal{E}$ as the addition of two matrices, $\mathcal{E}=\mathcal{T}+\mathcal{F}$, where $\mathcal{T}$ is the transition matrix and $\mathcal{F}$ is the infection matrix, which are given by

$$
\mathcal{T}=\left(\begin{array}{cccccc}
T & -a_{2}^{\alpha} I & -a_{3}^{\alpha} I & \cdots & -a_{k-1}^{\alpha} I & -a_{k}^{\alpha} I \\
I & 0 & 0 & \cdots & 0 & 0 \\
O & I & 0 & \cdots & 0 & 0 \\
\vdots & \vdots & \vdots & & \vdots & \vdots \\
0 & 0 & 0 & \cdots & I & 0
\end{array}\right) \text { and } \mathcal{F}=\left(\begin{array}{cccc}
F & 0 & \cdots & 0 \\
O & 0 & \cdots & 0 \\
O & 0 & \cdots & 0 \\
\vdots & \vdots & & \vdots \\
0 & 0 & \cdots & 0
\end{array}\right)
$$

in such a way that the matrix $T$ is the diagonal matrix formed by the diagonal of matrix $\bar{E}$ and $F=\bar{E}-T$. Then, we define the $(\alpha, k)$-Basic Reproduction Number $((\alpha, k)-\mathrm{BRN}), R_{0}(\alpha, k)$, as

$$
R_{0}(\alpha, k)=\rho\left(\mathcal{F}(I-\mathcal{T})^{-1}\right)
$$

Next result gives an explicit expression of $(\alpha, k)-$ BRN.

Proposition 5. The $(\alpha, k)-B R N$ of the DTFO system (16) is

$$
R_{0}(\alpha, k)=\sqrt{\frac{\beta \sigma P}{\left(1-q+\Sigma_{k}^{\alpha}\right)\left(1-s+\Sigma_{k}^{\alpha}\right)}} .
$$

Proof. Firstly, we show that the matrix $I-\mathcal{T}$ is an invertible matrix proving that $\rho(\mathcal{T})<1$. The characteristic polynomial of matrix $\mathcal{T}$ is given by

$$
|\lambda I-\mathcal{T}|=\left|\lambda^{k} I-\lambda^{k-1} T+\lambda^{k-2} a_{2} I+\cdots+\lambda a_{k-1} I+a_{k} I\right|=0,
$$

where we have used that the matrix $T$ is stable and $I-T$ is an invertible matrix since $\alpha \in \Lambda_{q, s}$.

By the structure of the matrices involved in the above equation, we only need to study the following system of equations

$$
\begin{gathered}
\lambda^{k}-(q-1+\alpha) \lambda^{k-1}+a_{2} \lambda^{k-2}+\cdots+a_{k-1} \lambda+a_{k}=0 \\
\lambda^{k}-(s-1+\alpha) \lambda^{k-1}+a_{2} \lambda^{k-2}+\cdots+a_{k-1} \lambda+a_{k}=0 .
\end{gathered}
$$

In a similar way as it is done in the proof of Proposition 4, we take into account that $|\lambda|<1$ for any solution of both equations. Then, we have proved that $\rho(\mathcal{T})<1$ and the matrix $I-\mathcal{T}$ is invertible.

Next, we have to calculate the product $\mathcal{F}(I-\mathcal{T})^{-1}$. Since the matrix $\mathcal{F}$ is a block matrix with a unique non-null block, we only need the corresponding block of the inverse matrix. Splitting $(I-\mathcal{T})^{-1}$ into blocks with the corresponding sizes of the blocks of $\mathcal{F}$ we have

$$
(I-\mathcal{T})^{-1}=\left(\begin{array}{ll}
B_{1} & B_{2} \\
B_{3} & B_{4}
\end{array}\right)
$$


Note that, in this case, $R_{0}(\alpha, k)=\rho\left(F B_{1}\right)$.

From the definition of inverse matrix and the structure of matrix $\mathcal{T}$ we have that

$$
\left(\begin{array}{cc}
I-T & X \\
Y & B_{b}
\end{array}\right)\left(\begin{array}{ll}
B_{1} & B_{2} \\
B_{3} & B_{4}
\end{array}\right)=I
$$

where $X=\left(\begin{array}{lll}a_{2} I & \cdots & a_{k} I\end{array}\right), Y=\left(\begin{array}{llll}-I & 0 & \cdots & 0\end{array}\right)^{T}$ and

$$
B_{b}=\left(\begin{array}{ccc}
I & \cdots & 0 \\
-I & \cdots & 0 \\
\vdots & & \vdots \\
0 & \cdots I & 0 \\
0 & \cdots-I & I
\end{array}\right) .
$$

Solving the resulting matrix equations, we have that the block $B_{1}$ is

$$
B_{1}=\left(I-T+X B_{b}^{-1} Y\right)^{-1}=\operatorname{diag}\left(\frac{1}{1-q+\Sigma_{k}^{\alpha}}, \frac{1}{1-s+\Sigma_{k}^{\alpha}}\right) .
$$

Then, the eigenvalues of the matrix $F B_{1}$ are the solutions of the following equation

$$
\lambda^{2}-\frac{\beta \sigma P}{\left(1-q+\Sigma_{k}^{\alpha}\right)\left(1-s+\Sigma_{k}^{\alpha}\right)}=0 .
$$

So, the $(\alpha, k)$-Basic Reproduction Number is

$$
R_{0}(\alpha, k)=\sqrt{\frac{\beta \sigma P}{\left(1-q+\Sigma_{k}^{\alpha}\right)\left(1-s+\Sigma_{k}^{\alpha}\right)}} .
$$

Note that, in the particular case of $\alpha=1$, we have that $\Sigma_{k}^{1}=0$ and $R_{0}(1, k)=R_{0}$ where, $R_{0}$ is the Basic Reproduction Number of the system (1) (see [32]).

From [34,35], if $R_{0}(\alpha, k)<1$ then $\rho(\mathcal{E})<1$ and the system (25) is assymptotically stable to zero and it is unstable when $R_{0}(\alpha, k)>1$. Consequently, the disease-free equilibrium point of system (16) is assymptotically stable and unstable, respectively.

Using the expressions of the endemic equilibrium point (18) and the $(\alpha, k)-\mathrm{BRN}(29)$, we can analyze the stability of the disease-free equilibrium point, $P_{f}$, taking into account the size of the population $(P)$ of the model. Then, the following results follow.

Theorem 1. Given the DTFO system (16), if $P<x_{1 e}^{*}(\alpha, k)$ then $x_{f}^{*}$ is assymptotically stable and unstable if $P>x_{1 e}^{*}(\alpha, k)$.

In this theorem we have discussed if the disease-free equilibrium point is assymptotically stable. This leads us to introduce the lower bound $P_{l}(\alpha, k)=x_{1 e}^{*}(\alpha, k)$ and the set

$$
\Omega_{1}=\left\{x \in \Omega_{\alpha, k} / P<P_{l}(\alpha, k)\right\} .
$$

In the next result we study if this equilibrium point is globally assymptotically stable.

Theorem 2. If the solution of the DTFO system (16) is in $\Omega_{1}$, then the disease-free equilibrium point, $x_{f}^{*}$, is the unique equilibrium point of the system and it is globally assymptotically stable.

Proof. Since $P<P_{l}(\alpha, k)$, the $(\alpha, k)-$ BRN is less than $1, \rho(\bar{E})<1$ and the linear subsystem (25) is assymptotically stable to 0 . Now, we consider the last two equations of system (16). Taking into account that $x_{1}(t) \leq P$, $\forall t \geq 0$, we have that

$$
\left(\begin{array}{l}
x_{2}(t) \\
x_{3}(t)
\end{array}\right) \leq \bar{E} z(t)
$$

Then, we derive that

$$
0 \leq\left(\begin{array}{l}
x_{2}(t) \\
x_{3}(t)
\end{array}\right) \leq \bar{E}^{t} z(0) \quad \forall t \geq 0
$$

Using that $\rho(\bar{E})<1$, we have that $\lim _{t \rightarrow \infty} \bar{E}^{t} z(0)=0$ and, consequently, $\lim _{t \rightarrow \infty}\left(\begin{array}{l}x_{2}(t) \\ x_{3}(t)\end{array}\right)=0$. Since $x_{1}(t)+x_{2}(t)=P, t \geq 0$ we get $\lim _{t \rightarrow \infty} x_{1}(t)=P$.

Moreover, from $P<P_{l}(\alpha, k)$, the point $x_{f}^{*}$ is the unique equilibrium point of the system and it is globally assymptotically stable. 


\subsection{The dynamics of the endemic equilibrium point}

In this subsection we study the stability of the endemic equilibrium point, $x_{e}^{*}(\alpha, k)$, of the DFTO system (16). Thus, we are considering $R_{0}(\alpha, k)>1$, that is, the solution of the system is in $\Omega_{2}$, given by

$$
\Omega_{2}=\left\{x \in \Omega_{\alpha, k} / P_{l}(\alpha, k)<P\right\} .
$$

Theorem 3. Consider that the solution of the DTFO system (16) is in $\Omega_{2}$. If there exists $t_{0} \geq 0$ such that $x_{1}\left(t_{0}\right)<x_{1}\left(t_{0}-1\right)$ and $x_{3}\left(t_{0}+1\right) \geq x_{3}\left(t_{0}\right)$, then the endemic equilibrium point, $x_{e}^{*}(\alpha, k)$, is assymptotically stable.

Proof. First we prove the monotony of the successions $\left\{x_{1}(t)\right\}_{t \geq t_{0}},\left\{x_{2}(t)\right\}_{t \geq t_{0}}$ and $\left\{x_{3}(t)\right\}_{t \geq t_{0}}$. Since $x(t) \in \Omega_{2}, x_{1}(t) \geq 0$. So,

$$
\begin{aligned}
& x_{1}\left(t_{0}+1\right)-x_{1}\left(t_{0}\right)=\left(q-1+\alpha-\sigma x_{3}\left(t_{0}\right)\right) x_{1}\left(t_{0}\right)-\left(q-1+\alpha-\sigma x_{3}\left(t_{0}-1\right)\right) x_{1}\left(t_{0}-1\right)= \\
& =(q-1+\alpha)\left(x_{1}\left(t_{0}\right)-x_{1}\left(t_{0}-1\right)\right)-\sigma\left(x_{3}\left(t_{0}\right) x_{1}\left(t_{0}\right)-x_{3}\left(t_{0}-1\right) x_{1}\left(t_{0}-1\right)\right) .
\end{aligned}
$$

From condition $x_{3}\left(t_{0}+1\right) \geq x_{3}\left(t_{0}\right)$,

$$
\begin{aligned}
& x_{1}\left(t_{0}+1\right)-x_{1}\left(t_{0}\right)<(q-1+\alpha)\left(x_{1}\left(t_{0}\right)-x_{1}\left(t_{0}-1\right)\right)-\sigma x_{3}\left(t_{0}\right)\left(x_{1}\left(t_{0}\right)-x_{1}\left(t_{0}-1\right)\right)= \\
& =\left(q-1+\alpha-\sigma x_{3}\left(t_{0}\right)\right)\left(x_{1}\left(t_{0}\right)-x_{1}\left(t_{0}-1\right)\right)<0 .
\end{aligned}
$$

Then $x_{1}(t+1)<x_{1}(t), \forall t \geq t_{0}$.

Using that $x_{1}(t)+x_{2}(t)=P, \forall t \geq 0$, we obtain that $x_{2}(t+1)>x_{2}(t), \forall t \geq t_{0}$. Then, taking into account the third equation of system (16) we have that $x_{3}(t+1)>x_{3}(t), \forall t \geq t_{0}$.

Since $\left\{x_{3}(t)\right\}_{t \geq t_{0}}$ is an increasing succession and it is bounded, as we have seen in the proof of Proposition 4, the succession is convergent to the third component of the endemic equilibrium point. Analogously, we can do the same for $\left\{x_{1}(t)\right\}_{t \geq t_{0}}$ and $\left\{x_{2}(t)\right\}_{t \geq t_{0}}$ obtaining that they are convergent, respectively, to the first and second component of the endemic equilibrium point.

\section{Effects of the fractional order and the memory steps on the model behaviour}

As we have seen in the study of the system stability, it depends on the value of $(\alpha, k)-\mathrm{BRN}$. In this section, we are going to study the variation of $R_{0}(\alpha, k)$ depending on the value of the parameters $0<\alpha \leq 1$ and $k \geq 1$.

Clearly, the variation of the $(\alpha, k)-\mathrm{BRN}(29)$ depends on the variation of $\Sigma_{k}^{\alpha}$. For this last factor, we have the following result:

Proposition 6. Given $0<\alpha \leq 1$, the factor $\Sigma_{k}^{\alpha}$ satisfies

$$
\Sigma_{k}^{\alpha}<\Sigma_{k-1}^{\alpha}, \quad \lim _{k \rightarrow \infty} \Sigma_{k}^{\alpha}=0, \quad \text { and } \quad \lim _{\alpha \rightarrow 0} \Sigma_{k}^{\alpha}=1, \quad \forall k \geq 1 .
$$

Proof. From the definition of $\Sigma_{k}^{\alpha}$, given by expression (11), we have that

$$
\Sigma_{k}^{\alpha}=\Sigma_{k-1}^{\alpha}+a_{k}^{\alpha}<\Sigma_{k-1}^{\alpha}
$$

since $a_{j}^{\alpha}<0, \forall j \geq 1$, from Proposition 1. Moreover,

$$
\lim _{k \rightarrow \infty} \Sigma_{k}^{\alpha}=\lim _{k \rightarrow \infty}-\frac{k-\alpha}{\alpha} a_{k}^{\alpha}=\lim _{k \rightarrow \infty} \frac{(1-\alpha)(2-\alpha) \cdots(k-\alpha)}{k !}=0
$$

and $\lim _{\alpha \rightarrow 0} \Sigma_{k}^{\alpha}=1$ follows directly.

From the above proposition, definitions (29) and (11), the following results follow directly:

Proposition 7. Given $0<\alpha \leq 1$, the $(\alpha, k)-B R N$ (29) satisfies

(i) $\lim _{k \rightarrow \infty} R_{0}^{2}(\alpha, k)=\frac{\sigma \beta P}{(1-q)(1-s)}=R_{0}^{2}(1, k)$.

(ii) $R_{0}(\alpha, k)>R_{0}(\alpha, k-1), \quad \forall k \geq 1$

Analogously, for the case when a fixed $k$ is considered:

Proposition 8. Given $k \geq 1$, the $(\alpha, k)-B R N$ (29) satisfies

(i) $\lim _{\alpha \rightarrow 0} R_{0}(\alpha, k)=\frac{\sigma \beta P}{(2-q)(2-s)}$.

(ii) $R_{0}\left(\alpha_{1}, k\right)<R_{0}\left(\alpha_{2}, k\right) \quad \forall 0<\alpha_{1}<\alpha_{2}<1$. 
Table 2

Variation of $P_{l}(\alpha, k)$ in terms of $\alpha$ and $k$.

\begin{tabular}{llllllll}
\hline$k / \alpha$ & 0.8 & 0.82 & 0.84 & 0.86 & 0.88 & 0.9 & 1 \\
\hline 2 & 48.40 & 42.52 & 37.17 & 32.33 & 27.96 & 24.03 & 10 \\
3 & 35.34 & 31.39 & 27.83 & 24.63 & 21.75 & 19.18 & 10 \\
4 & 29.03 & 26.03 & 23.34 & 20.93 & 18.78 & 16.86 & 10 \\
5 & 25.32 & 22.89 & 20.71 & 18.77 & 17.03 & 15.49 & 10 \\
6 & 22.88 & 20.82 & 18.98 & 17.34 & 15.89 & 14.59 & 10 \\
\hline
\end{tabular}

Table 3

Variation of $R_{0}^{2}(\alpha, k)$ in terms of $\alpha$ and $k$.

\begin{tabular}{lllllll}
\hline$k / \alpha$ & 0.8 & 0.82 & 0.84 & 0.86 & 0.88 & 0.9 \\
\hline 2 & 0.52 & 0.59 & 0.67 & 0.77 & 0.89 & 1.04 \\
3 & 0.71 & 0.80 & 0.89 & 1.01 & 1.15 & 1.30 \\
4 & 0.86 & 0.96 & 1.07 & 1.19 & 1.33 & 1.48 \\
5 & 0.98 & 1.09 & 1.20 & 1.33 & 1.47 & 1.61 \\
6 & 1.09 & 1.20 & 1.32 & 1.44 & 1.57 & 1.71 \\
\hline
\end{tabular}

Note that these properties can be extended to the endemic equilibrium point having that the susceptible individuals for this point grow when $k$ grows keeping $\alpha$ constant and decreases when $\alpha$ grows for a given value of $k$. That is:

$$
\frac{(2-q)(2-s)}{\sigma \beta}>\ldots>x_{1 e}^{*}(\alpha, k)>x_{1 e}^{*}(\alpha, k+1)>\ldots>\lim _{k \rightarrow \infty} x_{1 e}^{*}(\alpha, k)=\frac{(1-q)(1-s)}{\sigma \beta},
$$

and

$$
\lim _{\alpha \rightarrow 0} x_{1 e}^{*}(\alpha, k)=\frac{(2-q)(2-s)}{\sigma \beta}>\ldots>x_{1 e}^{*}\left(\alpha_{1}, k\right)>x_{1 e}^{*}\left(\alpha_{2}, k\right)>\ldots>x_{1 e}^{*}(1, k)=\frac{(1-q)(1-s)}{\sigma \beta},
$$

with $0<\alpha_{1}<\alpha_{2} \leq 1$.

\section{Numerical examples}

To study the fractional order model with $k$ steps of memory numerically, we consider the system (15), with the condition of constant population $P=x_{1}(t)+x_{2}(t)$,

$$
\begin{aligned}
& \left.x_{1}(t+1)=(q-1+\alpha)\right) x_{1}(t)-\sum_{j=2}^{k} a_{j}^{\alpha} x_{1}(t+1-j)-\sigma x_{1}(t) x_{3}(t)+P\left(1-q+\Sigma_{k}^{\alpha}\right), \\
& x_{3}(t+1)=(s-1+\alpha) x_{3}(t)-\sum_{j=2}^{k} a_{j}^{\alpha} x_{3}(t+1-j)-\beta x_{1}(t)+\beta P .
\end{aligned}
$$

This model corresponds to a cubicle of an animal farm, where there is an infectious disease, which is transmitted by indirect contact. That is, Susceptible individuals $x_{1}(t)$, are infected by contact with a Contaminant, $x_{3}(t)$, coming from Infected animals and staying in the environment [3]. The following parameters are considered: $q=0.9, \sigma=10^{-5} \mathrm{Bacteria}^{-1}, \beta=10^{2}$ Bacteria.Indiv $^{-1}$ colony-forming unit (c.f.u.) and $s=0.9$.

First, we study the dependence on the fractional order $\alpha$ and the memory step $k$ of the bound $P_{l}(\alpha, k)=x_{1 e}^{*}(\alpha, k)$ given by Theorem 1 that measures the maximum population to assure that the system is asymptotically stable to the disease-free equilibrium point. The behaviour of this bound is presented in Table 2 .

Now, we set the population $P=25$, which is a representative value of the results presented in Table 2 . Computing the square of the $(\alpha, k)-$ BRN for this population, we obtain the results presented in Table 3 .

We observe that for a given value of $\alpha$ we find models that are stable to the desease-free equilibrium point and other models that are unstable, depending on the memory steps $k$ considered.

With the population $P=25$ and the fractional order $\alpha=0.82$, the evolution of the susceptible individuals, $x_{1}(t)$ considering $k=3,4,5$ and 6 is shown in Fig. 1. It is observed that for $k=3$ and $4 x_{1}(t)$ tends to the disease-free equilibrium point, while for $k=5$ and 6 it tends to the endemic equilibrium point, as it is stated in Theorem 3. Moreover, we have that the susceptible population decreases when the number of steps $k$ increases. In this figure we have also plotted (in black) the evolution of $x_{1}(t)$ for the standard integer case $\alpha=1$, which is independent of the value of the step $k$ since this model does not have memory. We can observe that the susceptible population is smaller in the case $\alpha=1$ than for $\alpha=0.82$. That is, in the case $\alpha=0.82$ there is less infection than for the model with $\alpha=1$.

Finally, to show that depending on the population size the model can have negative solutions we set the total population to $P=270$, which is a value larger than $P_{u}(\alpha, k)$ given by (19), consider $\alpha=0.82$ and $k=5$ and 6 . The evolution of $x_{1}(t)$ in both cases is shown in Fig. 2. It is observed that when $k=5$ the solution remains nonnegative but for $k=6$ the model provides negative values of $x_{1}(t)$ presenting this solution an oscillating behaviour, with no biological sense. 


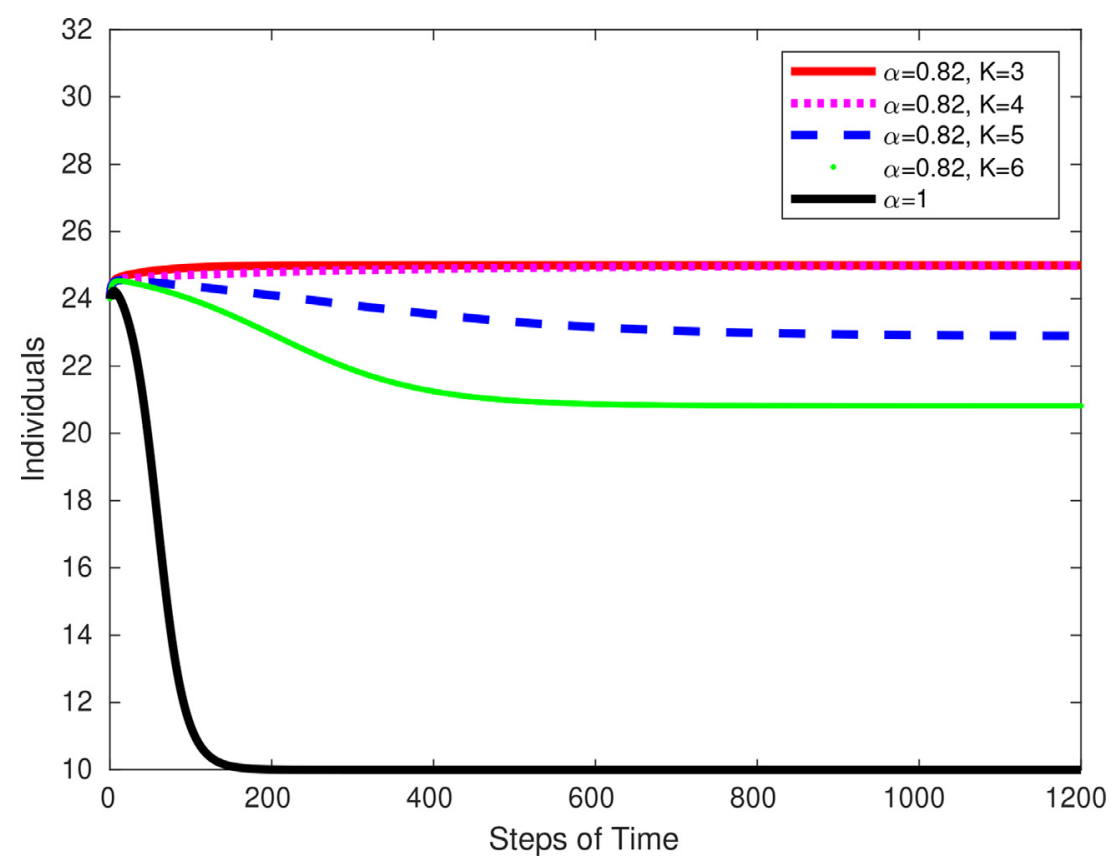

Fig. 1. Evolution of $x_{1}(t)$ when $\alpha=0.82$ and $k=3,4,5$ and 6 with a population $P=25$. The evolution of $x_{1}(t)$ for $\alpha=1$, which is independent of $k$, is represented in black.

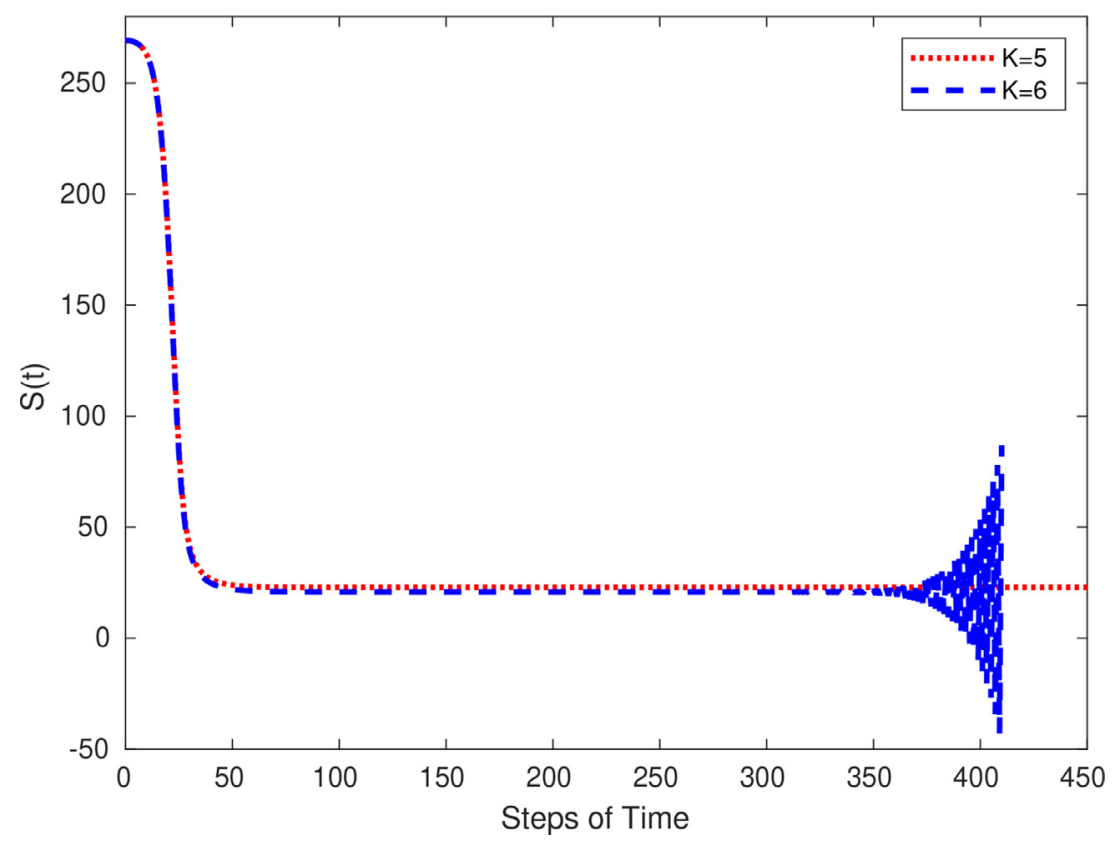

Fig. 2. Evolution of $x_{1}(t)$ when $\alpha=0.82$ and $k=5$ and 6 when $P=270$.

\section{Conclusions}

A mathematical model that represents the evolution of a disease through indirect transmission has been considered and a discrete fractional order model with $k$ memory steps is proposed. The $(\alpha, k)$-Basic Reproduction Number has been constructed as a function of the fractional order, $\alpha$, and memory steps, $k$.

The stability of the equilibrium points of the model has been analyzed in terms of the $(\alpha, k)$-Basic Reproduction Number and the population size. State admissible sets have been constructed in order to assure different situations: a nonnegative solution, that the disease disappears or that the disease remains near an endemic equilibrium point. An exhaustive anal- 
ysis of the $(\alpha, k)$-Basic Reproduction Number and the endemic equilibrium point has been performed depending on the fractional order and the memory steps considered.

A qualitative analysis of the behaviour of the model solution is given using numerical examples for different fractional orders and different number of memory steps. Also, it is shown that if we take some parameters out from the admissible sets, the biological sense of the model is lost since the solution can become negative. In future researches, it would be interesting to deep into this loss of the nonnegativity constraint of the solution by comparing this situation with other discrete or continuous models.

It is left as an open question to obtain more information about how to discriminate in a real case between the behavior of the standard model with $\alpha=1$ and the fractional order models with $\alpha<1$. For this, the inverse problem has to be considered. That is, we have to start from a set of observations, which includes the prevalence of a certain disease and the amount of contaminant, at different times, and look for determining the parameters of the model that provide a best fit to available observations. For this, efficient algorithms have to be designed because the dependence of the fractional model on the parameters $\alpha$ and $k$ is very involved.

\section{Acknowledgments}

The authors would like to thank the editor and referees for their valuable comments, which helped to improve the manuscript.

\section{References}

[1] F. Brauer, Z. Shuai, P. van den Driessche, Dynamics of an age-of-infection cholera model, Math. Biosci. 10 (5) (2013) 1335-1349, doi:10.3934/mbe.2013. 10.1335.

[2] B. Cantó, C. Coll, E. Sánchez, Stabilization of an epidemic model via n-periodic approach, J. Appl. Math. Comput. Sci. 28 (1) (2018) 185-195, doi:10. 2478/amcs-2018-0014.

[3] C. Coll, E. Sánchez, Epidemic spreading by indirect transmission in a compartmental farm, Appl. Math. Comput. 386 (2020), doi:10.1016/j.amc.2020. 125473.

[4] H.E. Emmert, L.S.J. Allen, Population persistence and extinction in a discrete-time, stage-structured epidemic model, J. Differ. Equ. Appl. 10 (2004) 1177-1199, doi:10.1080/10236190410001654151.

[5] I. Podlubny, Fractional differential equations: An Introduction to Fractional Derivatives, Fractional Differential Equations, to Methods of their Solution and Some of Their Applications, Elsevier, 1998.

[6] M. Caputo, Linear models of dissipation whose q is almost frequency independent II, Geophys J Int 13 (5) (1967) 529-539, doi:10.1111/j.1365-246X. 1967.tb02303.x.

[7] G. Wu, D. Baleanu, Discrete fractional logistic map and its chaos, Nonlinear Dyn. 75 (2014) 283-287, doi:10.1007/s11071-013-1065-7.

[8] G. Wu, D. Baleanu, S. Zeng, et al., Discrete fractional diffusion equation, Nonlinear Dyn. 80 (2015) 281-286, doi:10.1007/s11071-014-1867-2.

[9] J.H. He, A tutorial review on fractal spacetime and fractional calculus, Int. J. Theor. Phys. 53 (11) (2014) 3698-3718.

[10] I. Podlubny, Geometric and physical interpretation of fractional integration and fractionl differentation, Fractional Calculus and Applied Analysis 5 (4) (2002) 367-386.

[11] R. Pakhira, U. Ghosh, S. Sarkar, Study of memory effects in an inventory model using fractional calculus, Appl Math Sci. 12 (17) (2018) 797-824, doi:10.12988/ams.2019.9111.

[12] R.L. Stanislavsky, Memory effects and macroscopic manifestation of randomness, Phys. Rev. E 61 (5) (2008) 4752-4759, doi:10.1103/PhysRevE.61.4752.

[13] Y. Enatsu, Y. Nakata, Y. Muroya, Global stability for a discrete SIS epidemic model with immigration of infectives, J. Differ. Equ. Appl. 18 (2012) $1913-$ 1924, doi:10.1080/10236198.2011.602973.

[14] R.M. May, Biological populations with non-overlapping generations: stable points, stable cycles and chaos, Science 186 (4164) (1974) 645-647, doi:10. $1126 /$ science.186.4164.645.

[15] L.J. Allen, Some discrete-time SI, SIR, and SIS epidemic models, Math. Biosciences 124 (1994) 83-105, doi:10.1016/0025-5564(94)90025-6

[16] N. Hernández-Cerón, Z. Feng, C. Catillo-Chavez, Discrete epidemic models with arbitrary stage distributions and applications to disease control, Bull. Math. Biol. 75 (2013) 1716-1746, doi:10.1007/s11538-013-9866-X.

[17] H. Cao, H. Tan, The discrete tuberculosis transmission model with treatment of latently infectes individuals, Adv. Differ. Equ. 165 (2015), doi:10.1186 s13662-015-0505-8.

[18] M.A.M. Abdelaziz, A.I. Ismail, F.A. Abdullah, M.H. Mohd, Bifurcations and chaos in a discrete SI epidemic model with fractional order, Adv. Differ. Equ. 44 (2018), doi:10.1186/s13662-018-1481-6.

[19] M.A.M. Abdelaziz, A.I. Ismail, F.A. Abdullah, M.H. Mohd, Codimension one and two bifurcations of a discrete-time fractional order SEIR measles epidemic model with constant vaccination, Chaos Soliton Fract. 144 (2020) 110104, doi:10.1016/j.chaos.2020.110104.

[20] I. Ameen, P. Novati, The solution of fractional order epidemic model by implicit adam methods, App. Math. Model. 43 (2017) 78-84, doi:10.1016/j.apm. 2016.10.054.

[21] Y. Ding, H. Ye, A fractional-order differential equation model of HIV infection of CD4+ t-cells, Math. Comp. Model. 50 (2009) 386-392, doi:10.1016/j. mcm.2009.04.019.

[22] J. Huo, H. Zhao, Dynamical analysis of a fractional SIR model with birth and death on heterogeneous complex networks, Physica A 448 (2016) 41-56, doi:10.1016/j.physa.2015.12.078.

[23] S.R. Saratha, G.S.S. Krishnan, M. Bagyalakshmi, Analisys of a fractional epidemic model by fractional generalised homotopy analysis method using modified riemann-lioville derivative, Appl. Math. Model. 92 (2021) 525-545, doi:10.1016/j.apm.2020.11.019.

[24] A. Dzielinski, D. Sierociuk, Stability of discrete fractional order state-space systems, Procceding of 2-IFAC Workshop on Fractional Differentiation and its Applications, Porto, Portugal, July 19-21, 2006.

[25] S. Guermah, S. Djennoune, M. Bettayeb, A new approah for stability analysis of linear discrete-time fractional-order systems, New Trends in Nanotechnology and Fractional Calculus Applications 10.1007/978-90-481-3293-5_11.

[26] T. Kaczorek, Practical stability of positive fractional discrete-time linear systems, Bull. Polish Acad. Sci. Tech. Sci. 56 (4) (2008) 313-317, doi:10.2478/ amcs-2018-0034.

[27] T. Kaczorek, Selected Problems of Fractional Systems Theory, 411, Berlin, Springer, 2011.

[28] F.A. Rihan, Delay Differential Equations and Applications to Biology, Berlin, Springer, 2021.

[29] F. Brauer, A new epidemic model with indirect transmission, J. Bio. Dyn. 11 (2017) 285-293. 10.108/17513758.2016.1207813

[30] Z. Lu, Y.T. Grohn, R.L. Smith, J.S. Karns, E. Hovingh, Y.H. Schukken, Stochastic modeling of imperfect salmonella vaccines in an adult dairy herd, Bull. Math. Biol. 76 (2014) 541-565, doi:10.1007/s11538-013-9931-5. 
[31] K. Prévost, P. Magal, C. Beaumont, A model of salmonella infection whitin industrial house hens, J. Theo. Biol. 242 (2006) 755-763, doi:10.1016/j.jtbi. 2006.04.019.

[32] B. Cantó, C. Coll, E. Sánchez, Epidemic dynamics of an infection through the pathogen density in the environment, CR. Acad. Bulg. Sci. 69 (7) (2016) $835-844$.

[33] R.I. Joh, H. Wang, H. Weiss, J.S. Weitz, Dynamics of indirectly transmitted infectious diseases with immunological threshold, B. Math. Biol. 71 (2009) 845-862, doi:10.1007/s11538-008-9384-4.

[34] C.K. Lie, H. Schneider, Application of perron-frobenius theory to population dynamics, J. Math. Biol. 44 (2002) 450-462, doi:10.1007/s002850100132.

[35] L.J.S. Allen, P. van den Driessche, The basic reproduction number in some discrete-time epidemic models, J. Diff. Eq. App. 14 (10-11) (2008) 1127-1147, doi:10.1080/10236190802332308. 